\title{
UNIQUENESS AND THE FORCE FORMULAS FOR PLANE SUBSONIC FLOWS
}

\author{
BY \\ ROBERT FINN AND DAVID GILBARG
}

Introduction. The first proof of uniqueness of a plane subsonic flow of a compressible fluid past an obstacle was given by Bers [1]. This proof utilizes an elaborate mathematical apparatus encompassing some of the most advanced tools of modern function theory. A conceptually simpler proof under somewhat weaker hypotheses and a proof of the Joukowsky force formula, due to the authors [3], make essential use of a general existence theorem and hence cannot properly be called elementary. In this note we show that the uniqueness of a compressible flow and the Joukowsky force formula can be obtained directly from a simple geometrical property of the velocity field defined by the flow. Specifically, we base our proof on the facts that the velocity components $u, v$ of the flow define a quasi-conformal( $\left.{ }^{1}\right)$ mapping of the $(x, y)$ plane, and that a quasi-conformal mapping with dilatation ratio not exceeding $\kappa=1 / \mu$ satisfies at each point a Hölder condition with exponent $\mu$. Until recently we would have considered this proof more difficult than our original one. We are, however, now able to refer to the preceding paper [5], in which a simple demonstration is given for the needed lemma. We note, further, that a simpler form of the arguments in [5] suffices for the present paper.

The problem of proving uniqueness of a flow past an obstacle $P$ can be divided into two parts; to prove the uniqueness if the velocity at infinity and circulation are both prescribed, and to prove that if $P$ has a corner or cusp $T$ the circulation is uniquely determined by the condition that the speed is finite at $T$. We shall settle both points here, but for the second we need the additional assumption, not used in [1] or [3], that the velocity components have bounded derivatives up to $P$. (This assumption can be avoided by a discussion analogous to that in $[3, \S 6.2]$.) By the same method we shall also obtain a new proof, simpler than that of [3], for the force formula of gas dynamics.

1. Uniqueness with prescribed circulation. As in [3] we define the velocity potential of a flow past $P$ to be a solution $\phi(x, y)$ of

$$
\left(\rho \phi_{x}\right)_{x}+\left(\rho \phi_{y}\right)_{y}=0
$$

where $\rho=f\left(\phi_{x}^{2}+\phi_{y}^{2}\right)=f\left(q^{2}\right)$, and we call the flow subsonic if $\rho+2 f^{\prime} q^{2}>0$. We assume this condition satisfied for all $q$ such that $0 \leqq q<q_{c r} \leqq \infty$. The functions

Received by the editors November 30, 1956. $[5]$.

(1) For the definition of this and other terms appearing here we refer the reader to [3] and 
$u=\phi_{x}$ and $v=\phi_{y}$ are called the velocity components of the flow. We assume the flow to be directed tangentially $(\partial \phi / \partial n=0)$ on $P$.

Equation (1) is an integrability condition for a stream function $\psi(x, y)$ such that

$$
\psi_{y}=\rho \phi_{x}, \quad-\psi_{x}=\rho \phi_{y} .
$$

For a flow past $P, \psi=$ const. on $P$.

Suppose we are given two subsonic flows $(\phi, \psi),(\bar{\phi}, \bar{\psi})$ past $P$ for which the velocities $(u, v)$ and $(\bar{u}, \bar{v})$ tend to a common subsonic limit $\left(u_{1}, v_{0}\right)$ at infinity and for which the circulations are the same,

$$
\Gamma=\oint d \phi=\oint d \bar{\phi}
$$

for any closed curve surrounding $P$. For a region $\varepsilon_{r}$ bounded by $P$ and by a circumference $C_{r}$ of radius $r$ enclosing $P$, we consider the identity

$$
\int_{\mathcal{E}_{r}}\left(\begin{array}{ll}
\Phi & \Psi \\
x & y
\end{array}\right) d x d y=\int_{C_{r}} \Phi d \Psi
$$

where $\Phi=\phi-\bar{\phi}, \Psi=\psi-\bar{\psi}$, and the integrand on the left is the Jacobian of the mapping $(\Phi, \Psi)$. (Note that by (3) this mapping is 1-valued.)

The Jacobian has constant sign in $\varepsilon_{r}$ and vanishes only if $\Phi_{x}^{2}+\Phi_{\nu}^{2}=0$. For set $\rho \phi_{x}=Q(u, v), \rho \phi_{y}=B(u, v)$ and consider the function

$$
\begin{aligned}
F(t)= & \Phi_{x}\{\propto[\bar{u}+t(u-\bar{u}), \bar{v}+t(v-\bar{v})]-\propto[\bar{u}, \bar{v}]\} \\
& +\Phi_{y}\{\Theta[\bar{u}+t(u-\bar{u}), \bar{v}+t(v-\bar{v})]-\Theta[\bar{u}, \bar{v}]\}, \quad 0 \leqq t \leqq 1 .
\end{aligned}
$$

Then $F(0)=0$ and $F(1)$ is the given Jacobian. But $F^{\prime}(t)$ is, by the assumption that both flows are subsonic, a definite quadratic form in $\Phi_{x}, \Phi_{y}$.

Our problem is thus to show that the line integral in (4) tends to zero as $r \rightarrow \infty$. To do this we consider first the behavior at infinity of the velocity fields of each of the given flows. Using (1) we find that $(u, v)$ appears as a solution of the system

$$
a u_{x}+2 b u_{y}+c v_{y}=0, \quad u_{y}-v_{x}=0
$$

where $a=\rho+2 \rho^{\prime} u^{2}, b=2 \rho^{\prime} u v, c=\rho+2 \rho^{\prime} v^{2}$. From this follows that for any subsonic flow, the complex function $w=u-i v$ defines a quasi-conformal mapping of the $(x, y)$ plane. By a linear transformation of $(x, y)$ we may arrange that the dilatation ratio tends to one at infinity, and by continuity of $a, b, c$, this ratio will be arbitrarily close to one in the exterior of $C_{r}$ if $r$ is chosen sufficiently large. Hence, using Theorem 1 of [5], we find the existence of a constant $C(\epsilon)$ such that $\left|w-w_{0}\right|<C r^{-1}$ for any given $\epsilon>0$. Therefore $|w-\bar{w}|<2 C r^{\epsilon-1}$ as $r \rightarrow \infty$. Integrating this inequality, we find $|\Phi|=|\phi-\bar{\phi}|$ $<C_{1} r^{\epsilon}$ for some constant $C_{1}$. But we have seen that 


$$
\left(\begin{array}{ll}
\Phi & \Psi \\
x & y
\end{array}\right)
$$

can be expressed as a definite quadratic form in $\Phi_{x}, \Phi_{y}$. Hence $\Phi+i \Psi$ defines a quasi-conformal mapping in the exterior of $P$. Let $\kappa$ be the maximum dilatation ratio of this mapping in some neighborhood of infinity. Applying Theorem 4 of [5], we see that if $\epsilon$ is chosen smaller than $1 / \kappa$ the singularity of $\Phi+i \Psi$ at infinity must be removable. In particular $\Phi$ tends to a limit at infinity and since we are free to adjust $\Phi$ by an additive constant we may assume $\Phi \rightarrow 0$. Further, for any $\epsilon>0$ there is a constant $C(\epsilon)$ such that $\Phi<C r^{\epsilon-1}$. Thus,

$$
\left|\oint_{C_{r}} \Phi d \Psi\right| \leqq \text { const. } \oint_{C_{r}}\left(\Psi_{x}^{2}+\Psi_{y}^{2}\right)^{1 / 2} d s \cdot r^{\epsilon-1} \text {. }
$$

But $\Psi_{x}=\rho u-\bar{\rho} \bar{u}=\rho(u-\bar{u})+(\rho-\bar{\rho}) \bar{u}=(u-\bar{u})(\rho+\bar{u} \partial \rho / \partial u)+(v-\bar{v}) \bar{u} d \rho / d v$, where $\partial \rho / \partial u$ and $\partial \rho / \partial v$ are to be evaluated at certain intermediate values of their arguments, and a similar relation holds for $\Psi_{y}$. Thus, using the above estimate for $|w-\bar{w}|$, we see that for any prescribed $\epsilon>0$ there is a constant $C(\epsilon)$ such that $\left(\Psi_{x}^{2}+\Psi_{y}^{2}\right)^{1 / 2}<C r^{\epsilon-1}$. Inserting this result into (5) leads directly to the desired estimate on the line integral and completes the uniqueness proof. We formulate our result:

THEOREM 1. A subsonic flow past a profile is uniquely determined by its free stream velocity and circulation.

2. The Kutta-Joukowski condition. We assume now that $P$ has a continuously turning tangent with the exception of a trailing edge $T$ at which the direction of the tangent has a simple discontinuity of amount $k \pi, 0<k \leqq 1$. We assume further that there is a neighborhood $N_{T}$ of $T$ in which each point of $P$ can be contacted from the exterior by a circle which meets no other point of $P$ and that the radius of all such circles can be chosen bounded from zero in $N$. Finally we suppose that the velocity components of the flow have uniformly bounded derivatives in $N_{T}$. Under these conditions we obtain the analogue of the result of Kutta [8] and Joukowski [9]:

THEOREM 2. The circulation is uniquely determined by the velocity at infinity.

Proof. Suppose to the contrary, that there exist two flows $(\phi, \psi),(\bar{\phi}, \bar{\psi})$ with the same limiting velocity and different circulation $\Gamma \neq \bar{\Gamma}$. For the function $\Phi+i \Psi=(\phi-\bar{\phi})+i(\psi-\bar{\psi})$ we find easily that after a linear transformation of the $(x, y)$ plane (we do not change notation),

$$
\Phi_{x}^{2}+\Phi_{y}^{2}+\Psi_{x}^{2}+\Psi_{y}^{2} \leqq 2 K\left(\begin{array}{ll}
\Phi & \Psi \\
x & y
\end{array}\right)
$$


where $K \rightarrow 1$ as $O\left(r^{\epsilon-1}\right)$ when $r \rightarrow \infty$ for any $\epsilon>0$. If we set $\tilde{\phi}+i \tilde{\psi}=\Phi+i \Psi$ $+i(\Gamma-\bar{\Gamma}) \log z / 2 \pi$ then $\tilde{\phi}+i \tilde{\psi}$ will be a single valued function of $(x, y)$ in a neighborhood of infinity, hence also in a neighborhood of the origin in the $\xi+i \eta$ plane, where $\xi+i \eta=1 /(x+i y)$. The inequality (6) transforms to

$$
\tilde{\phi}_{\xi}^{2}+\tilde{\phi}_{\eta}^{2}+\tilde{\psi}_{\xi}^{2}+\tilde{\psi}_{\eta}^{2} \leqq 2 K\left(\begin{array}{ll}
\tilde{\phi} & \tilde{\psi} \\
\xi & \eta
\end{array}\right)+O\left(r^{-1+\epsilon}\right)
$$

where $r$ now denotes $\left(\xi^{2}+\eta^{2}\right)^{1 / 2}$. Following the proof of Theorem 1 we see that $\tilde{\phi}+i \tilde{\psi}=\mathrm{O}\left(r^{-\epsilon}\right)$ as $r \rightarrow 0$. Thus $\tilde{\phi}+i \tilde{\psi}$ satisfies the hypotheses of Theorem 4 in [5] and we conclude that $\tilde{\phi}+i \tilde{\psi}$ tends to a limit at infinity in the $(x, y)$ plane. But this means $\Psi \rightarrow-\infty$ as $x^{2}+y^{2} \rightarrow \infty$. On the other hand, an application of the mean value theorem (cf. [2, pp. 274-277]) shows that $\Psi$ satisfies a partial differential equation of the form

$$
\alpha \Psi_{x x}+2 \beta \Psi_{x y}+\gamma \Psi_{y y}+\sigma \Psi_{x}+\tau \Psi_{y}=0
$$

where $\alpha, \beta, \gamma$ are continuous up to $P, \alpha \gamma-\beta^{2}>0$, and $\sigma, \tau$ are bounded in $N_{T}$ and continuous throughout the exterior of $P$. It is known [6] that a solution of such an equation admits no maximum or minimum interior to its region of definition. Since $\Psi=0$ on $P$, we conclude $\Psi<0$ in the exterior of $P$.

We may now apply a lemma of E. Hopf [7]: at all points of $P$ in $N_{T}$ except at the point $T, \partial \Psi / \partial n<-\delta<0$, where $n$ denotes the exterior directed normal. Since $\partial \phi / \partial n=\partial \bar{\phi} / \partial n=0$ on $P$, this means $\rho \partial \phi / \partial s>\bar{\rho} \partial \bar{\phi} / \partial s+\delta$ for an appropriate choice of positive sense for the arc length $s$ on $P$. Because both flows are subsonic we conclude $\partial \phi / \partial s>\partial \phi / \partial s+\delta^{\prime}$ for some $\delta^{\prime}>0$. But by assumption the velocity of each flow is continuous up to $T$, hence on opposite sides of $T$ on $P$ each velocity must be directed in opposite senses with respect to $s$ (if $k=1$ ) or else tend to zero at $T$. This contradicts the preceding inequality and establishes Theorem 2 .

3. The force on $P$. The classical expressions for the components of the net force exerted by the fluid on $P$ are

$$
\begin{aligned}
& X=-\int_{0}^{2 \pi}[p \cos \theta+\rho u(v \sin \theta+u \cos \theta)] r d \theta, \\
& Y=-\int_{0}^{2 \pi}[p \sin \theta+\rho v(v \sin \theta+u \cos \theta)] r d \theta
\end{aligned}
$$

where the integrations are performed over a circumference $C_{r}$ of radius $r$ enclosing $p$ and the pressure $p$ has the form

$$
p=\text { const. }-\int \rho q d q \text {. }
$$

We shall take (7) as definition.

From the proof of Theorem 1 we know that

$$
u=u_{0}+O\left(r^{-1+\varepsilon}\right), \quad v=O\left(r^{-1+\varepsilon}\right)
$$


where we have introduced a rotation of coordinates so that $v_{0}=0$. From these estimates we find

$p=$ const. $-\rho u\left(u-u_{0}\right)+O\left(r^{-2+2 \epsilon}\right), \quad \rho u=u_{0} \rho+\rho_{0} u-u_{0} \rho_{0}+O\left(r^{-2+2 \epsilon}\right)$.

Thus,

$$
X=-u_{0} \int_{0}^{2 \pi} \rho(v \sin \theta+u \cos \theta) r d \theta+O\left(r^{-1+2 \epsilon}\right) .
$$

The integral appearing here represents the flux of fluid crossing $C_{r}$ in unit time and hence vanishes (this is easily proved formally, using (1)). Since $\epsilon$ is an arbitrary positive quantity, we may choose $\epsilon<1 / 2$ and let $r \rightarrow \infty$ to obtain

$$
X=0 .
$$

Similarly

$$
Y=-\rho_{0} u_{0} \int_{0}^{2 \pi}(v \cos \theta-u \sin \theta) r d \theta+O\left(r^{-1+2 \epsilon}\right),
$$

which implies

$$
Y=-\rho_{0} u_{0} \Gamma .
$$

These formulas give the extension to plane compressible flows of the D'Alembert paradox and the Joukowski lift formula of incompressible flow. For a corresponding result in three dimensions we refer the reader to [4].

\section{REFERENCES}

1. L. Bers, Existence and uniqueness of a subsonic flow past a given profile, Communications on Pure and Applied Mathematics vol. 7 (1954) pp. 441-504.

2. R. Courant and D. Hilbert, Mathematische Physik II, Berlin, Julius Springer, 1937.

3. R. Finn and D. Gilbarg, Asymptotic behavior and uniqueness of plane subsonic flows, Communications on Pure and Applied Mathematics vol. 10 (1957) pp. 23-63.

4. - Three-dimensional subsonic flows, and asymptotic estimates for elliptic partial differential equations, Acta Math. vol. 98 (1957) pp. 265-296.

5. R. Finn and J. Serrin, On the Hölder continuity of quasi-conformal and elliptic mappings, Trans. Amer. Math. Soc. (to appear).

6. E. Hopf, Elementäre Bemerkungen über die Lösungen partieller Differentialgleichungen zweiter Ordnung vom elliptischen Typus, Sitzungsber. Berl. Akademie der Wissenschaften und der Literatur vol. 19 (1927) pp. 147-152.

7. - A remark on linear elliptic differential equations of the second order, Proc. Amer. Math. Soc. vol. 3 (1952) pp. 791-793.

8. W. M. Kutta, Sitzb. d. k. bayr. Akademie der Wissenschaften und der Literatur, 1910.

9. N. Joukowski, Aérodynamique, Trans. by Drzewiecki, Paris, Gauthier-Villars, 1916.

California Institute of Technology, Pasadena, Calif.

INDIANA UNIVERSITY, BLOOMINGTON, IND. 